\title{
Between a rock and a hard place: adaptive sensing and site-specific dispersal
}

\author{
Bethany Nichols ${ }^{1}$, Gerhard Leubner-Metzger ${ }^{1}$, and Vincent A A Jansen ${ }^{2}$ \\ ${ }^{1}$ Royal Holloway University of London \\ ${ }^{2}$ Royal Holloway, University of London
}

April 28, 2020

\begin{abstract}
Environmental variability can lead to dispersal: why stay put if it is better elsewhere? Without clues about local conditions, the optimal strategy is often to disperse a set fraction of offspring. Many habitats contain environmentally differing subhabitats. Is it adaptive for individuals to sense in which sub-habitat they find themselves, using environmental clues, and respond plastically by altering the dispersal rates? This appears to be done by some plants which produce dimorphic seeds with differential dispersal properties in response to ambient temperature. Here we develop a mathematical model to show, that in highly variable environments, not only does sensing promote plasticity of dispersal morph ratio, but individuals who can sense their sub-habitat and respond in this way have an adaptive advantage over those who cannot. With a rise in environmental variability due to climate change, our understanding of how natural populations persist and respond to changes has become crucially important.
\end{abstract}

\section{Introduction}

Environmental conditions are ever-changing, temporally and spatially. Trying to survive amid a change in conditions within a local habitat requires species to adapt and develop specialised mechanisms. In many cases, however, the changes are to such an extent that it is better to leave altogether, be it for one or more generations, or altogether. In these cases, many species have adapted to disperse. Dispersal can be defined as the movement of an individual from site of birth to site of reproduction. If better sites can be reached via dispersal, this drives evolution as organisms escape unfavourable conditions (Matthysen, 2012). Dispersal can be divided into three phases: emigration, inter-patch movement and immigration. During emigration, the individual can obtain clues from both biotic and abiotic factors in the local sub-habitat. Using these clues, the individual can then either disperse or not. If they do, they enter the transfer phase or movement through the habitat, where many species require clues from the environment in order to choose a new location to settle in (Clobert et al. , 2001, 2012). The successful settlement into a new sub-habitat is the immigrant phase. Many species disperse passively, and so are unable to make informed choices about the final sub-habitat in which they find themselves.

Dispersal and emigration can reduce the likelihood of competition with kin, and mitigate against drift and inbreeding (Bengtsson, 1978; Wolff, Lundy and Baccus, 1988; Perrin and Mazalov, 1999; Ronce, 2007; Hidalgo, De Casas and Muñoz, 2016). However, environmental variability is arguably the most important driver for dispersal evolution. Many species have offspring that either can or cannot disperse. Experimental research has demonstrated that the ratio of dispersing to non-dispersing offspring changes in response to the environment; For example, Sinervo et al. demonstrated the interaction between maternal environmental conditions, and the resulting offspring dispersal ratio in lizards (Sinervo et al. , 2006). Dispersal plasticity is 
taxonomically widespread and similar results are seen in animals, insects and plants (Harrison, 1980; Fox and Mousseau, 1998; Dingle, 2006; King and Roff, 2010; Steiner et al. , 2012; Arendt, 2015; Duckworth, Belloni and Anderson, 2015). The ability to alter offspring dispersal ratio is a selective advantage when persisting in highly variable environments (Arendt, 2015).

Within a habitat, there are often several sub-habitats. These sub-habitats can differ in biotic and abiotic factors. Environmental variability is determined by how much and how frequently these factors fluctuate. When environmental conditions within sub-habitats are constant, but the sub-habitats differ in quality, a nondispersal strategy is optimal. This is because offspring dispersing from the native sub-habitat will encounter lower quality sub-habitats more often than higher quality sub-habitats (Hastings, 1983). However, in bethedging scenarios, if the environment fluctuates, with sites of differing quality across time, but statistically the same overall quality, then producing some dispersing offspring is optimal (Harper, 1977; Den Boer, 1981; Venable and Brown, 1993; Baskin and Baskin, 1998; Starrfelt and Kokko, 2012). If the environmental conditions in sub-habitats fluctuate and their quality is overall statistically different, it is possible that dispersal rates are sub-habitat specific (McPeek and Holt, 1992). But when sub-habitats are of more or less equal quality, yet differ in variability, what dispersal strategies will evolve? Will dispersal be adaptive, and if so, will the dispersal rates be habitat specific (Seale and Nakayama, 2019)?

In mountainous habitats, the sub-habitat at the top of the mountain can be considerably different from the sub-habitat at the bottom of the mountain. If neither sub-habitat is of substantially higher quality, and environmental variability is different within each, is sensing and site-specific dispersal adaptive? Here, we use the term "site-specific dispersal" to describe altering the ratio of dispersing to non-dispersing offspring produced by an individual, in response to the environment that they experience during their lifetime. An example of such site-specific dispersal is given by the plant Aethionema arabicum. Ae. arabicum is an annual which grows along the steep stony slopes of the Anatolian Mountains, at a range of 0-3000 meters above sea level (Bhattacharya et al. , 2019). Ae. arabicum exhibits fruit and seed dispersal dimorphism and is able to undergo site-specific dispersal between sub-habitats of similar quality but different variability by sensing its location through temperature (Fig. 1) (Lenser et al. , 2016; Mohammadin et al. , 2017; Arshad et al. , 2019; Seale and Nakayama, 2019).

Mountainous habitats, including the Anatolian Mountains, can be roughly divided into two sub-habitats: high elevation, and low elevation (Velchev, 1984; Mohammadin et al. , 2017). Neither sub-habitat is favourable, neither are optimal. The higher elevation is dry, exposed, and rocky, making the abiotic conditions unfavourable, however, there is little to no competition. At low elevation, the environment is overcrowded, shaded and highly competitive, providing many biotic stresses. However, the constant availability of water supply and nutrients (Atalay, 2006). One sub-habitat is environmentally variable in terms of abiotic stresses but with few competition stresses, while the other is environmentally constant but much more over-crowded, making them of both low quality and differing in variability.

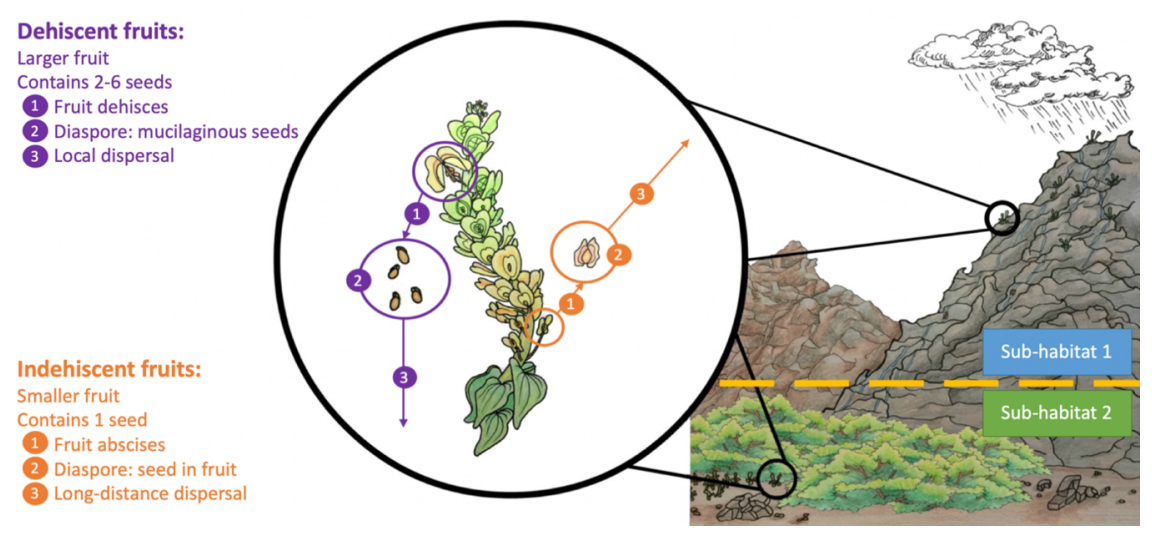


Fig. 1: Aethionema arabicum sub-habitats and dimorphic dispersal strategy (not to scale). Aethionema arabicum is a dimorphic plant species that grows along steep stony slopes in the Anatolian Mountains and produces a ratio of dispersing and non-dispersing offspring, dependent on the ambient temperature of the native sub-habitat (Lenser et al. , 2016).

Distributing offspring across sub-habitats can increase fitness and allows population survival in environments where they could not without dispersal (Jansen and Yoshimura, 1998). If an organism can sense which subhabitat it resides in, is it adaptive to have dispersal strategies that are contingent on the sub-habitat? Here we show theoretically how individuals altering their dispersal ratio in response to localised environmental variability could be aided through sensing. We develop a theoretical approach for sub-habitats that differ in their environmental variability. We show that site-specific dispersal ratios, aided by sensing, are adaptive in response to differences between sub-habitats, and therefore there is selection for traits that possess this ability.

\section{Results}

\section{The model}

Dispersal models have been used to describe the implications of dispersal on the fitness of species that persist within a single habitat (Cohen, 1966, 1967; Fretwell and Lucas, 1970; Bulmer, 1984; Cohen and Levin, 1991), and dispersal ratios have been fixed (Hastings, 1983). Although, in some of these models, multiple sub-habitats are considered through the dispersal into or out of the stated sub-habitat, the models are not equipped to investigate the impact of dispersing into and out of multiple sub-habitats at once.

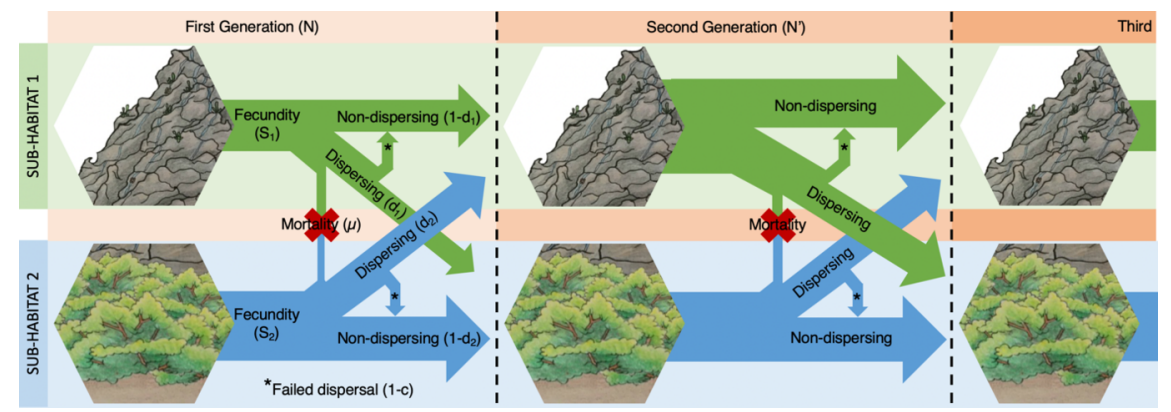

Fig. 2: Mathematical model of dispersal across two sub-habitats of differing environmental variability. The model is divided into two sub-habitats: 1 and 2. Each sub-habitat has a starting population of either the same or different sizes. The arrows branch-off to demonstrate the fate of the offspring: a proportion will not disperse, and the rest will. Of those that disperse, some will fail to disperse, and some will die before reaching the other sub-habitat. In each generation, sub-habitat 2 remains constant in the number of offspring produced and therefore, the dispersing to non-dispersing ratio is constant. Sub-habitat 1, on the other hand, experiences environmental variability.

Here, we constructed a matrix model to describe dispersal in two sub-habitats: 1 and 2 (Fig. 2). The model gives the number of individuals that will be in each sub-habitat in the following generation. The model describes an annual plant, and so there will be no overlapping generations, and that dispersal is not a cognitive decision, but it can be informed by environmental clues. The number of offspring/fecundity of each plant can be the same or different in each sub-habitat. There are two variables for dispersal, one for each habitat. This allows for the rate at which the offspring disperse from either sub-habitat to be altered (Fig. 2). The diagramatic model in figure 2 is representative of the matrix model used in this paper:

$N^{\prime}=$ 


$$
\left[\begin{array}{cc}
1-d_{1}(1-c)\left(S_{1}+\left(\frac{v}{f}\right)\right) & a m p ;(1-\mu) d_{2} S_{2}(1-c) \\
(1-\mu) d_{1}\left(S_{1}+\left(\frac{v}{f}\right)\right)(1-c) & \text { amp } ;\left(1-d_{2}(1-c)\right) S_{2}
\end{array}\right] \text { N. (1) }
$$

The model is divided into sub-habitats 1 and 2, each starting with a population of size $\mathrm{N}_{\mathrm{x}}$. The arrows branch-off to demonstrate the fate of the offspring $\left(S_{1}\right)$ : a proportion will not disperse $\left(1-d_{x}\right)$, and the rest will $\left(\mathrm{d}_{\mathrm{x}}\right)$. Of those that disperse, some will fail to disperse and remain in the native sub-habitat (1-c), where they will attempt leave the native sub-habitat but remain in the native sub-habitat, and some will die before reaching the other sub-habitat $(\mu)$. In each generation, sub-habitat 2 remains constant in the number of offspring produced and therefore, the dispersing to non-dispersing ratio is constant. Sub-habitat 1, on the other hand, experiences environmental variability, with a severity (v), which fluctuates the number of offspring produced (fecundity) depending on whether the environment is good or bad in that year, and the frequency in which there are good years $\left(\frac{1}{f}\right)$, and so the number of offspring differ year-on-year. The matrix demonstrated above is for good years. In bad years, the fecundity is $\left(S_{1}-\left(1-\left(\frac{1}{f}\right)\right) v\right)$. This is represented by the width of the arrows. The pattern of variability is changed for each simulation. The fitness of the population was measured as the long-term average population growth rate.

We assume that sub-habitat 2 has a constant, homogenous environment. Sub-habitat 1 experiences a range of differently variable environments. We define environmental variability by 2 variables: frequency and severity of "bad years". Productive years, when fecundity is high, are noted as "good years". Frequency is measured by the number of bad years between good years. In a bad year, only a percentage of the offspring produced in a good year will survive. This percentage is determined by the severity variable.

\section{Sensing and site-specific dispersal rates}

When the environment fluctuates within a sub-habitat, and environmental variability is sufficiently high, the optimal dispersal strategy is to produce a proportion of dispersing and non-dispersing seeds (Fig 3). If individuals do not have clues about which habitat they are in, then the only way to do this is disperse from all habitats in the same rate (combinations of dispersal rates that are the same in both patches sit on the diagonal in Figs 3a and b). However, when such clues are available and individuals can get a sense as to where they are, they adjust the dispersal rate to each habitat. The optimal dispersal strategy in both examples is then away from the diagonal, providing selection for site-specific dispersal rates, and, in this way, there is selection for sensing and adapting the dispersal rate to the native habitat. Which dispersal rates are selected for depends on the details, such as changes in frequency of environmental variability. Here we show the difference when there is a change in the frequency of the good years.
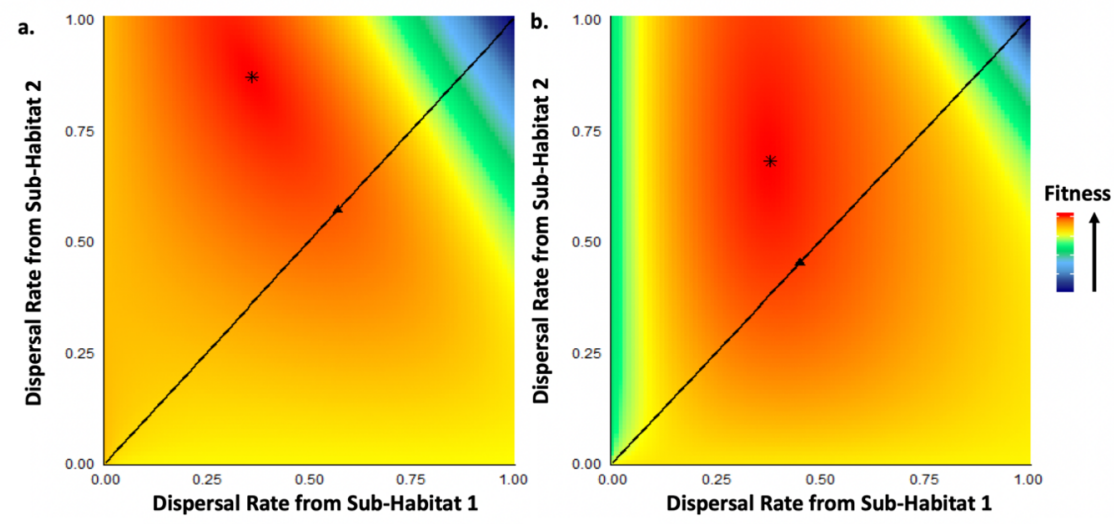

Fig. 3: Optimising dispersal strategy with changing frequency of bad years. Figures $3 a$ and $3 b$ differ in frequency of good years. $3 a$ has a frequency of $\frac{1}{3}$ and $3 b$ has a frequency of $\frac{1}{5}$. The heat maps 
demonstrate the fitness (growth rate) of the population at different dispersal ratios. indicates the optimal dispersal ratio to maximise fitness. It is mostly assumed in dispersal models that sub-habitats have used the same dispersal rate for all habitats, and so this is indicated by the black line where the dispersal rate from sub-habitat 1 is equal to the dispersal rate from sub-habitat 2. indicates the optimal dispersal ratio along this line. In both, there is additional fitness when you move away from the black line and have a dispersal rate dependent on the sub-habitat and the frequency of variability. Variables used to generate graphs: $v=20, S 1$ $=18, S 2=14, f$ in $3 a=3, f$ in $3 b=5$.

By how much the two sub-habitats differ in fecundity is critical for contingent dispersal strategies to evolve. Consider two sub-habitats. In sub-habitat 1 , the environment is highly variable, with fecundity being high in intermittently good years, and low in bad years. Sub-habitat 2 has a constant environment. In Figure 4, the fecundity in sub-habitat 2 is put on a sliding scale. If the average in sub-habitat 2 is higher than that of sub-habitat 1, then the dispersal rate from sub-habitat 2 will be 0 and sub-habitat 1 will be left empty. The optimal dispersal rate from sub-habitat 1 can then take any value (Fig. 4, region 3, see supplementary material). If the average fecundity in sub-habitat 1 is much higher than sub-habitat 2 the reverse happens and the dispersal rate from sub-habitat 1 is zero and sub-habitat 2 is left empty. When, however, fecundity in the sub-habitats are roughly the same, the optimum strategy is to have contingent dispersal rates so the that the offspring is distributed over the two sub-habitats: one with high variability and is very productive in good years, and one with low variability but is lowly productive, causing both to be of similar fitness. There is no obvious best location, and in some years one sub-habitat will be better, in other years the other. In environments such as this, site-specific, non-zero dispersal rates will evolve. In order to demonstrate this, we completed a sensitivity analysis which modulated the model parameters. Figure 5 shows the result of modulating the parameter $\mu$. Further results can be found in the supplementary material.

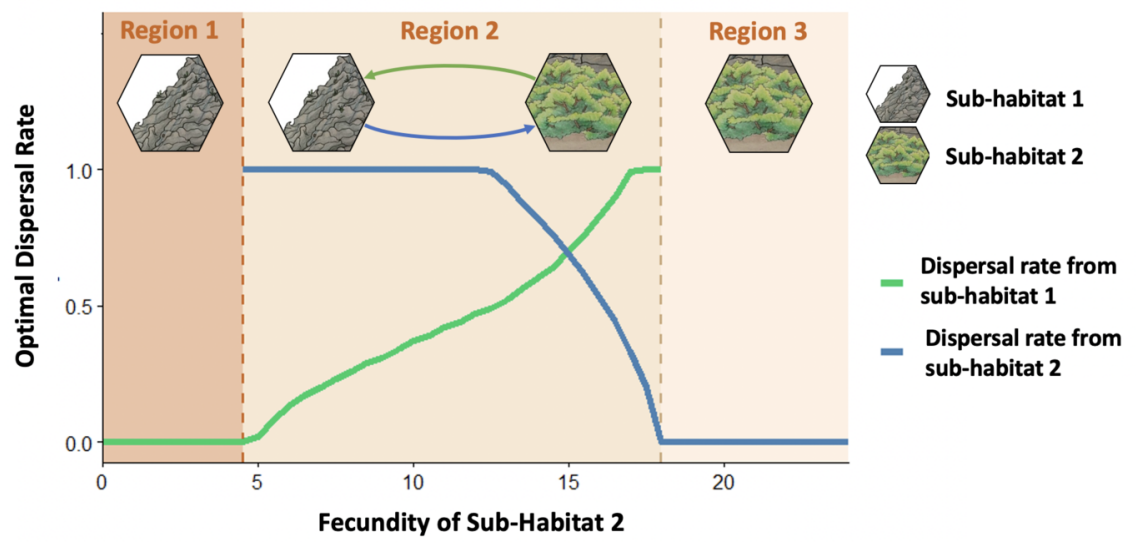

Fig. 4: Optimising dispersal strategy for changes in fecundity. The optimal dispersal rate from sub-habitats 1 and 2 is dependent on the relationship between the average fecundity of sub-habitats 1 and 2. At low average fecundities of sub-habitat 2, the optimal sub-habitat is sub-habitat 1 and so dispersal will favour remaining in sub-habitat 1. The converse is true at high average fecundities of sub-habitat 2 . When the average fecundities of sub-habitats 1 and 2 are about equal, mixed dispersal ratios are seen. Under these conditions, there is additional fitness in sensing the environment in which the individual has grown and producing site-specific dispersal ratios in response. Variables used: $v=21, S 1=18, S 2=0-23, f=5, \mu$ $=0, c=0$.

When the severity ( $\mathrm{v}$ ) of the fluctuations in sub-habitat 1 is low, the region in which sensing is adaptive is small, however, as $\mathrm{v}$ increases, the region widens (Fig. 5a). By plotting the width of region 2 from Figure 4 with an increase of $\mathrm{v}$, the effect of the severity of fluctuations in sub-habitat 1 can be seen. With an increase in $\mathrm{v}$ comes an increase in the width of region 2 . The more severe the fluctuations, the bigger the 
scope for site-specific dispersal rates. In this way, as the fluctuations become more severe, there is a greater potential for site-specific dispersal rates to evolve. When the fluctuations are small, the effect is negligible. This is more apparent with an increase in dispersal mortality (Fig. 5b). At low severity, there is no scope for site-specific dispersal. With high severity, site-specific dispersal creates additional fitness and in order to produce site-specific rates, a mechanism for sensing will evolve. This demonstrates the importance of severity as a driver for the evolution of sensing and site-specific dispersal.

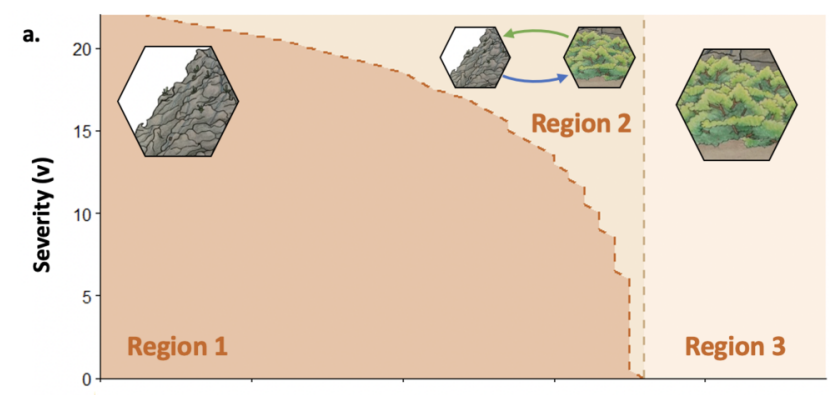

b.

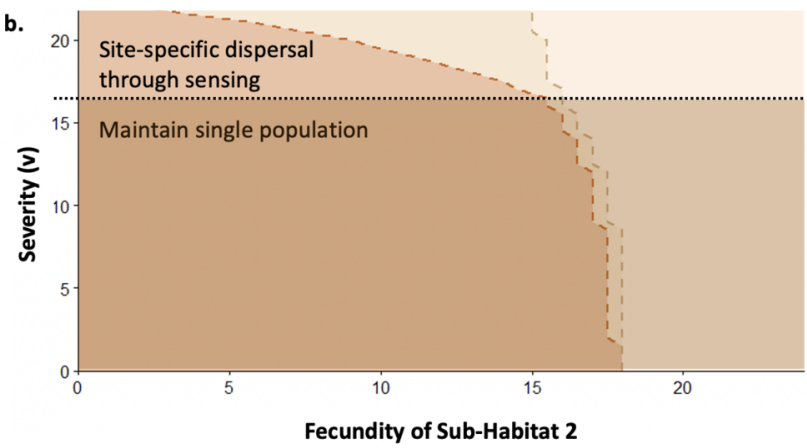

Sub-habitat 1

Sub-habitat 2

Fig. 5: Evolution of sensing and site-specific dispersal with increasingly severe environmental variability. By mapping the size of the regions in Figure 4 with a change in severity ( $v)$, it is apparent that with an increase in $v$, there is greater potential for site-specific dispersal to evolve. With an increase in $\mu$ (fig. 5b), the potential for site-specific dispersal to evolve is limited to high values of $v$, demonstrating the importance of $v$ as a driver for this effect. Variables used: $v=0-23, S 1=18, S 2=0-23, f=5, c=0$, $\mu$ in $5 a=0, \mu$ in $5 b=0.1$.

\section{Discussion}

MacArthur asks, "Why would any individual ever migrate to a less favourable area? Why not stay put if it is better at home?" (MacArthur, 1972). Often times the environmental variability of each sub-habitat is unique and different from the others within the habitat. When this is the case, there is additional fitness if individuals have the ability to alter the dispersal rate from each habitat. This means that having site-specific dispersal rates is adaptive (Fig. 3). When environmental variability within each sub-habitat is considered, and individuals who are able to sense their location have an additional fitness, they benefit from the ability to alter their dispersal ratio in accordance with where they find themselves.

Populations existing within a widespread habitat will be subjected to different environmental conditions depending on where within that habitat they find themselves. To what degree can organisms have information about where they find themselves? Across some habitats, there are likely to be differences in environmental conditions, which will have a bearing on the fitness of a population. For instance, differences in temperature or rainfall will vary and can be used as clues to which sub-habitat an organism finds itself in. Plants and seeds, for instance, sense changes in temperature through the seasons and use these as cues to define their timing 
of flowering, germination and seedling emergence (Linkies et al. , 2010). Germination timing is controlled via dormancy cycling for which temperature and moisture are the two most important environmental cues (Finch-Savage and Footitt, 2017). A typical plant has over 600 receptor-like kinases (RLKs) involved in sensing specific molecules including from the environment; animals evolved only about $1 \%$ of this (Shiu and Bleecker, 2001). Although plants do not have a nervous system, they can sense their environment extremely well and integrate the environmental factors both long-term and short-term. Similar cues are used by the individual seed or plant to determine where they might find themselves within their habitat. Using these cues, individuals are able to produce site-specific offspring ratios, such as the example of Ae. arabicum (Lenseret al. , 2016).

Many species, including plant species, are limited to sensing in the emigration phase of dispersal where other species are able to also sense their environment during the transfer phase. In this way, they cannot sense the surrounding sub-habitats and make informed dispersal choices about the sub-habitat into which they ultimately immigrate. As a result, many species depend on the environmental clues in the emigration phase and so the risk of dispersing is much higher, because they cannot make an informed decision once they have dispersed. For this reason, it is crucial to sense the condition of the local environment to determine if the risk of entering a potentially worse sub-habitat is worth taking the chance. Adding the interactions between dispersal mechanisms and environmental conditions into models, such as the one presented in this paper, gives a fuller, more in-depth understanding into population dynamics and the consequences of the environment on dispersal (Seale and Nakayama, 2019).

If the future quality of the entire environment is highly predictable, it is possible to use cues to determine the next future state. In term of dispersal, the best strategy is then to somehow disperse offspring to the best sub-habitat. Often times, the future is uncertain and information about possible future states is unavailable. What we show here that, theoretically, even if that is the case, systematic differences between sub-habitats, be it either in the average quality or in its variability, can lead to adaptations that favour site-specific dispersal, provided the organism can get clues as to which sub-habitat they find themselves in. How they sense the environment is unimportant, so long as it provides information to help them determine in which sub-habitat they are in. In the case of Ae arabicum, for instance, the key difference between the high and low altitude environments might be in terms of competition and severity and unpredictability. There is no need to sense these factors directly: temperature, even though in itself potentially not the most important factor, gives a clue as to the likely altitude the organism finds itself and can therefore be used as a clue. Sensing provides the individual with enough information about the local environment to determine where they are, and site-specific dispersal allows the individual to alter their dispersal ratio in order to maximise the likelihood of the offspring surviving to the following year.

The importance of dispersal plasticity in response to local environmental variability is something seen across the kingdoms (Arendt, 2015). Poa alpina is an alpine species that persists in a highly variable environment. The species adopts a seed-reproduction strategy at lower elevations, and bulbil-reproduction strategy at higher elevations (Steiner et al. , 2012). This allows it to occupy a large ecological niche so that it can persist in multiple sub-habitats in case one or more of these sub-habitats become unfavourable. In many insect species, wing polymorphism dictates dispersal rates. In aphids, offspring are either winged or wingless, making them dispersing or non-dispersing. Competition, crowding and host condition appear to be the driving forces for the ratio of offspring dimorphism, all of which the maternal aphid is able to sense and respond to by producing wingless or winged offspring (Harrison, 1980). Cycles of bluebird species have been shown to be driven by maternal effects. Following the creation of a new environment by a wildfire, the area is colonised by mountain bluebirds (Sialia currucoides ). To compete with the mountain bluebirds, maternal western bluebirds (Sialia mexicana) produce aggressive, dispersing offspring. Once the area is colonised by western bluebirds, nonaggressive, non-dispersing offspring are produced. Population density, resource limitations and competitive interactions all appear to be cues for maternal western bluebirds (Duckworth, Belloni and Anderson, 2015).

The habitat in which Ae. arabicum exists in the Anatolian Mountains can be roughly divided into two 
sub-habitats: high elevation, and low elevation (Velchev, 1984; Mohammadin et al. , 2017). At high elevation, the environment is dry, exposed, and rocky, with little to no competition. The exposure makes it prone to extreme weather conditions, and so the environmental variability is considered to be higher. At low elevation, the environment is overcrowded, shaded and highly competitive, with better availability of a steady water supply and nutrients. At this elevation, the environment is more competitive but generally sheltered, making the environment less variable year on year (Atalay, 2006). As well as this, there is also a temperature gradient along the elevation, as there is a drop of $3^{\circ} \mathrm{C}$ for each 300 metres above sea level (masl) climbed. Aethionema arabicum has been found growing between 0-3000 masl (Bhattacharya et al. , 2019). By sensing the environment into which the dispersing, indehiscent fruits (IND) disperse through this temperature gradient, the resultant plant grows and produces different ratios of dispersing to non-dispersing offspring (Lenser et al. , 2016; Arshad et al. , 2019).

The life-history of Ae. arabicum fits with the strategy observed in the model. There is a dramatic temperature difference along the elevation of the slopes on which they grow (Fig. 1 in Arshad et al., 2016 (Arshad et al. , 2019)). Aethionema arabicum has been shown to alter the ratio of IND and non-dispersing, dehiscent fruits $(\mathrm{DEH})$ that it produces when the mother plant is grown at different temperatures during reproduction (Lenser et al. , 2016). In this way, the germination timing due to temperature differences (season, elevation) and the consequently distinct temperature during plant growth and reproduction both influence the final offspring ratio of the plant (Lenser et al. , 2016; Arshad et al. , 2019).

In a lower temperature regime during reproduction, the plant produces more IND fruits. Therefore, it can be suggested that at higher altitudes, Ae. arabicum plants will produce more of the dispersing morphs. This would mean they are able to take advantage of the dispersing adaptations of the IND fruit: it's buoyant and aerodynamic nature. This would allow them to spread out across the mountain side in greater numbers and take advantage of wherever most is accommodating in the following year. At lower elevations, Ae. arabicum reduces the proportion of dispersal-type offspring with higher temperature and competition stress. However, in response to nutrient stress, Ae. arabicum increases the proportion of dispersal-type offspring (Bhattacharya et al. , 2019). Whether a plant emerges from an IND or DEH fruit does not seem to predict the ratio of dispersing to non-dispersing seeds that it will, in turn, produce. The plasticity is a response to the environmental conditions in which the plants are grown (Lenser et al. , 2016).

One way to efficiently alter the dispersal ratio is through heteromorphism. Heteromorphism was first described by Venable as "the production by single individuals of seeds of different form or behaviour" (Venable, 1985). In this context, behaviour refers to their ecological traits such as their dispersal mechanism. There is a fitness advantage of being able to detect location and therefore, evolving a method to respond by dispersing out of or remaining within the environment is crucial for many species. The main method of doing so for many species is heteromorphism: producing two or more offspring phenotypes that have no, or different methods of dispersal (Imbert, 2002).

One such model to describe the evolution of dispersal heteromorphism is by Venable (Venable, 1985). In this model, the production of two seed morphs in different year types is investigated. The two morphs have different mean and variance based on evolutionary constraints. Offspring morphs are adapted to perform in particular year types, causing more of one to be produced in its favourable year and more of the other to be produced in the opposing years. Evolutionary constraints between years lead to heteromorphism, which produce two offspring morphs that are better adapted to the evolutionary constraints. This is the generalised model used to describe the evolution of dispersal heteromorphism. Our model is an alternative that allows for severely fluctuating, multi-habitat environments. Furthermore, the model gives an explanation for the purpose of sensing in dispersal heteromorphism. Each individual produces two offspring morphs; neither is better adapted for a particular year type, but rather one type is able to disperse and the other is not. How much more or less the offspring disperse out of the sub-habitat is determined by where they are. In this way, our model shows the impact of difference in variability between multiple environments and how this leads to the evolution of sensing and site-specific dispersal plasticity.

Another method of responding to the environment is through responsive phenotype switching. This is 
where the individual senses an ambient environmental cue and switches its phenotype. However, this is costly because it relies on developing and maintaining machinery to detect environmental conditions. For organisms with a fast turnover rate, such as bacteria, switching rates that mimic the infrequent environmental variability can be favourable over sensing. This is called spontaneous stochastic switching. In environments where there is higher environmental variability, there is an additional benefit to responsive switching. On the other hand, if the environment is fairly constant and variability is infrequent or less, then the stochastic switching method is favoured, as the cost of sensory machinery is too high (Kussell and Leibler, 2005). This pattern mimics closely the pattern observed in our model.

In bet-hedging theory, geometric mean is used to describe fitness. Existing theories on bet-hedging assume that organisms respond to a single environmental variable, so that therefore the geometric mean of this variable can be used as a proxy for fitness. In an environment consisting of two distinct sub-habitats this is not possible as the growth rate of a population cannot be expressed as a simple geometric mean (Tuljapurkar, 1990). In this scenario, sensing doesn't evolve, because no one sub-habitat is better than any other, as they are all statistically identical, and therefore having site-specific dispersal rates gives no advantage. However, when the environments are statistically variable across the years, and multiple variables for population growth rate are introduced, as in our model, the need to sense location and produce site-specific dispersal rates is adaptive. Although bet-hedging within our model is possible, the results from our model go beyond bet-hedging theory (supplementary material).

Results from the model show that the dispersal strategy best adopted by individuals differs dramatically depending on the environmental variability between multiple sub-habitats. Previous models of dispersal have overlooked the importance of altering the dispersal ratio, depending on the many sub-habitats in which an individual may find itself. This "one size fits all" approach should be reconsidered, as it does not match the life history of species persisting in highly variable environments. Although it has been suggested that climate variability influences aphid reproduction being sexual or asexual by parthenogenesis, this has largely been overlooked in most other species (Halkett et al. , 2004).

A higher incidence of extreme weather conditions are on the rise as a result of climate change. Droughts, heatwaves, flash flooding, heavy downpour and hurricanes are just some of the unpredictable weather phenomena putting species at risk. Especially species that have evolved in lowly variable, temperate climates (Michener et al. , 1997; Watson et al. , 1998; Easterling et al. , 2000; McLaughlinet al. , 2002; Ummenhofer and Meehl, 2017). In the case of Ae. Arabicum, in a mountainous habitat, the two sub-habitats will experience climate change differently, due to their size and topography. Higher up mountains and on mountaintops, the environment is more exposed, and so will be more affected by windstorms and precipitation, where further down, the plains are generally sheltered, but prone to flooding and erosion (Barry, 1992; Beniston, 2006). Sub-habitats experiencing differing environmental stresses like this is also common in coastal areas (Keddy, 1981).

Invasive species also put ecosystems at risk. Phenotypic plasticity is an important adaptation to invasive species, as it allows them to occur in a wide range of environments (Richards et al. , 2006). With environmental change brings new opportunities for invasive species to disperse into such locations and outcompete native species. Dispersal strategies responding plastically to environmental changes are commonly researched in animals, but rarely are for plants (Imbert and Ronce, 2001). This is because plants have been widely regarded as passive organisms to those outside of plant sciences. However, it has been recognised that plants are able to undergo site-specific dispersal and can manipulate the dispersal phenotypes of their offspring in response to their environment (Seale and Nakayama, 2019). With little knowledge on how plasticity or lack thereof will affect plant populations in the wake of environmental unpredictability, this could put many species at risk.

Individuals alter the dispersal ratio of their offspring in response to localised environmental variability to an evolutionary advantage. When a species is dispersing between a rock and a hard place, the optimal strategy is to sense the environment and alter the dispersal ratio of their offspring in response. In this way, the population is balanced within a highly variable environment with multiple differing sub-habitats, 
where they would otherwise go extinct if the population was restricted to one sub-habitat. With increasingly severe fluctuations in environmental variability comes an increase for the scope of this dispersal strategy. Species adopting this lifestyle are able to thrive in otherwise challenging environments. However, dispersal plasticity is widely under researched and underappreciated across all kingdoms and with a rapid increase in environmental variability, one can only speculate the impact on the natural world.

\section{Methods}

The model (1) is comprised of four variables: number of plants in a site $\left(\mathrm{N}_{\mathrm{x}}\right)$, fecundity $\left(\mathrm{S}_{\mathrm{i}}\right)$ determined by how many seeds are produced by a single plant, dispersal rate $\left(\mathrm{d}_{\mathrm{i}}\right)$, and death rate $(\mu)$. The number of plants in a site in the following year $\left(N_{x}^{\prime}\right)$ is determined by the number of seeds that do not disperse $\left(1-d_{x} S_{x} N_{x}\right)$ plus the number of seeds that survive dispersal from the other site $\left(1-\mu d_{x} S_{x} N_{x}\right)$. The individuals are presumed to be annuals, and therefore do not survive into the following year. A proportion of seeds that disperse fail to do so and remain in the native sub-habitat. This percentage is denoted by $c$.

$$
N_{1}^{\prime}=1-d_{1}(1-c) S_{1} N_{1}+1-\mu d_{2}(1-c) S_{2} N_{2}
$$

$$
N_{2}^{\prime}=1-\mu d_{1}(1-c) S_{1} N_{1}+1-d_{2}(1-c) S_{2} N_{2}
$$

The model was then arranged to form a vector-matrix multiplication (2 and 3). Sub-habitat 2, is a constant environment so only experiences good years. Sub-habitat 1 fluctuates in environmental condition and the frequency of which it experiences good year is $\frac{1}{f}$. The proportion of $\mathrm{S}_{1}$ that survive, or the severity of the year, is $v$. In good years (2), the fecundity is increased by $\frac{v}{f}$ and in bad years (3), the fecundity is decreased by $\left(1-\left(\frac{1}{f}\right)\right) v$.

\section{Good Year:}

$N^{\prime}=$

$\left[\begin{array}{cc}\left(\left(1-d_{1}(1-c)\right)\left(S_{1}+\left(\frac{v}{f}\right)\right)\right. & a m p ;\left((1-\mu) d_{2} S_{2}\right)(1-c) \\ \left((1-\mu) d_{1}\left(S_{1}+\left(\frac{v}{f}\right)\right)\right)(1-c) & a m p ;\left(1-d_{2}(1-c)\right) S_{2}\end{array}\right] \mathrm{N}(2)$

\section{Bad Year:}

$$
\begin{aligned}
& N^{\prime}= \\
& {\left[\begin{array}{cc}
\left(1-d_{1}(1-c)\right)\left(S_{1}-\left(1-\left(\frac{1}{f}\right)\right) v\right) & a m p ;\left((1-\mu) d_{2} S_{2}\right)(1-c) \\
\left((1-\mu) d_{1}\left(S_{1}-\left(1-\left(\frac{1}{f}\right)\right) v\right)\right)(1-c) & a m p ;\left(1-d_{2}(1-c)\right) S_{2}
\end{array}\right] \mathrm{N}(3)}
\end{aligned}
$$

This model contains two variables for dispersal, $d_{1}$ and $d_{2}$. This is to allow for the dispersal rates between to the two sub-habitats to be altered independently. The model was run for 1000 iterations in $\mathrm{R}$, and the fitness $(Q)$ of the two sub-habitats was calculated at the end (4). Fitness was determined by the average growth rate of the population which was calculated by the change in population size divided by the number of simulations run.

$Q=\frac{\Delta N_{x}}{\Delta t_{x}}(4)$

The $\log$ of the average growth rate $(\log Q)$ was then calculated. $(\log Q)$ was then compared at different dispersal rates $\left(\mathrm{d}_{1}\right.$ and $\left.\mathrm{d}_{2}\right)$, and the relationship between the two investigated. For a detailed description of the model see the supplementary material.

\section{References}


Arendt, J. D. (2015) 'Effects of dispersal plasticity on population divergence and speciation', Heredity . Nature Publishing Group, 115(4), p. 306.

Arshad, W. et al. (2019) 'Dispersal biophysics and adaptive significance of dimorphic diaspores in the annual Aethionema arabicum (Brassicaceae)', The New phytologist . Wiley-Blackwell, 221(3), p. 1434.

Atalay, I. (2006) 'The effects of mountainous areas on biodiversity: a case study from the northern Anatolian Mountains and the Taurus Mountains', Grazer schriften der geographie und Raumforschung . Citeseer, 41, pp. $17-26$.

Barry, R. G. (1992) Mountain weather and climate . Psychology Press.

Baskin, C. C. and Baskin, J. M. (1998) Seeds: ecology, biogeography, and, evolution of dormancy and germination. 3rd edn. Elsevier.

Bengtsson, B. O. (1978) 'Avoiding inbreeding: at what cost?',Journal of Theoretical Biology . Elsevier, 73(3), pp. 439-444.

Beniston, M. (2006) 'Mountain weather and climate: a general overview and a focus on climatic change in the Alps', Hydrobiologia . Springer, 562(1), pp. 3-16.

Bhattacharya, S. et al. (2019) 'Naturally-primed life strategy plasticity of dimorphic Aethionema arabicum facilitates optimal habitat colonization', Scientific reports . Nature Publishing Group, 9(1), pp. 1-11.

Den Boer, P. J. (1981) 'On the survival of populations in a heterogeneous and variable environment', Oecologia . Springer, 50(1), pp. 39-53.

Bulmer, M. G. (1984) 'Delayed germination of seeds: Cohen's model revisited', Theoretical Population Biology . Elsevier, 26(3), pp. 367-377.

Clobert, J. et al. (2001) Dispersal .

Clobert, J. et al. (2012) Dispersal Ecology and Evolution . OUP Oxford.

Cohen, D. (1966) 'Optimizing reproduction in a randomly varying environment', Journal of theoretical biology . Elsevier, 12(1), pp. 119-129.

Cohen, D. (1967) 'Optimizing reproduction in a randomly varying environment when a correlation may exist between the conditions at the time a choice has to be made and the subsequent outcome', Journal of Theoretical Biology. Elsevier, 16(1), pp. 1-14.

Cohen, D. and Levin, S. A. (1991) 'Dispersal in patchy environments: the effects of temporal and spatial structure', Theoretical Population Biology. Elsevier, 39(1), pp. 63-99.

Dingle, H. (2006) 'Animal migration: is there a common migratory syndrome?', Journal of Ornithology . Springer, $147(2)$, pp. 212-220.

Duckworth, R. A., Belloni, V. and Anderson, S. R. (2015) 'Cycles of species replacement emerge from locally induced maternal effects on offspring behavior in a passerine bird', Science. American Association for the Advancement of Science, 347(6224), pp. 875-877.

Easterling, D. R. et al. (2000) 'Climate extremes: observations, modeling, and impacts', science . American Association for the Advancement of Science, 289(5487), pp. 2068-2074.

Finch-Savage, W. E. and Footitt, S. (2017) 'Seed dormancy cycling and the regulation of dormancy mechanisms to time germination in variable field environments', Journal of experimental botany. Oxford University Press UK, 68(4), pp. 843-856.

Fox, C. W. and Mousseau, T. A. (1998) 'Maternal effects as adaptations for transgenerational phenotypic plasticity in insects', Maternal effects as adaptations . Oxford University Press New York, 159, pp. 159-177. 
Fretwell, S. D. and Lucas, H. L. (1970) 'On territorial behaviour and other factors inftuencing habitat distribution in birds', Acta biotheor, 19, pp. 1-6.

Halkett, F. et al. (2004) 'Dynamics of production of sexual forms in aphids: theoretical and experimental evidence for adaptive "coin-flipping" plasticity', The American Naturalist . The University of Chicago Press, 163(6), pp. 112-125.

Harper, J. L. (1977) 'Population biology of plants.', Population biology of plants. Academic Press.

Harrison, R. G. (1980) 'Dispersal polymorphisms in insects', Annual Review of Ecology and Systematics . Annual Reviews 4139 El Camino Way, PO Box 10139, Palo Alto, CA 94303-0139, USA, 11(1), pp. 95-118.

Hastings, A. (1983) 'Can spatial variation alone lead to selection for dispersal?', Theoretical Population Biology . Elsevier, 24(3), pp. 244-251.

Hidalgo, J., De Casas, R. R. and Muñoz, M. (2016) 'Environmental unpredictability and inbreeding depression select for mixed dispersal syndromes', BMC evolutionary biology . BioMed Central, 16(1), p. 71.

Imbert, E. (2002) 'Ecological consequences and ontogeny of seed heteromorphism', Perspectives in Plant Ecology, Evolution and Systematics . Elsevier, 5(1), pp. 13-36.

Imbert, E. and Ronce, O. (2001) 'Phenotypic plasticity for dispersal ability in the seed heteromorphic Crepissancta (Asteraceae)', Oikos . Wiley Online Library, 93(1), pp. 126-134.

Jansen, V. A. A. and Yoshimura, J. (1998) 'Populations can persist in an environment consisting of sink habitats only', Proceedings of the National Academy of Sciences . National Acad Sciences, 95(7), pp. 36963698 .

Keddy, P. A. (1981) 'Experimental Demography of the Sand-Dune Annual, Cakile Edentula, Growing Along an Environmental Gradient in Nova Scotia', Journal of Ecology . [Wiley, British Ecological Society], 69(2), pp. 615-630. Available at: http://www.jstor.org/stable/2259688.

King, E. G. and Roff, D. A. (2010) 'Modeling the evolution of phenotypic plasticity in resource allocation in wing-dimorphic insects', The American Naturalist . The University of Chicago Press, 175(6), pp. 702-716.

Kussell, E. and Leibler, S. (2005) 'Phenotypic diversity, population growth, and information in fluctuating environments', Science. American Association for the Advancement of Science, 309(5743), pp. 2075-2078.

Lenser, T. et al. (2016) 'Developmental control and plasticity of fruit and seed dimorphism in Aethionema arabicum', Plant Physiology. Am Soc Plant Biol, 172(3), pp. 1691-1707.

Linkies, A. et al. (2010) 'The evolution of seeds', New Phytologist . Wiley Online Library, 186(4), pp. 817-831.

MacArthur, R. H. (1972) 'Geographical ecology', Harper and Row . New York, New York, USA.

Matthysen, E. (2012) 'Multicausality of dispersal: a review',Dispersal ecology and evolution . Oxford University Press Oxford, UK, 27, pp. 3-18.

McLaughlin, J. F. et al. (2002) 'Climate change hastens population extinctions', Proceedings of the National Academy of Sciences . National Acad Sciences, 99(9), pp. 6070-6074.

McPeek, M. A. and Holt, R. D. (1992) 'The evolution of dispersal in spatially and temporally varying environments', The American Naturalist . University of Chicago Press, 140(6), pp. 1010-1027.

Michener, W. K. et al. (1997) 'Climate change, hurricanes and tropical storms, and rising sea level in coastal wetlands',Ecological Applications . Wiley Online Library, 7(3), pp. 770-801.

Mohammadin, S. et al. (2017) 'Anatolian origins and diversification of Aethionema, the sister lineage of the core Brassicaceae', American journal of botany . Wiley Online Library, 104(7), pp. 1042-1054. 
Perrin, N. and Mazalov, V. (1999) 'Dispersal and inbreeding avoidance', The American Naturalist . The University of Chicago Press, 154(3), pp. 282-292.

Richards, C. L. et al. (2006) 'Jack of all trades, master of some? On the role of phenotypic plasticity in plant invasions',Ecology letters . Wiley Online Library, 9(8), pp. 981-993.

Ronce, O. (2007) 'How does it feel to be like a rolling stone? Ten questions about dispersal evolution', Annu. Rev. Ecol. Evol. Syst. Annual Reviews, 38, pp. 231-253.

Seale, M. and Nakayama, N. (2019) 'From passive to informed: mechanical mechanisms of seed dispersal', New Phytologist. Wiley Online Library.

Shiu, S. and Bleecker, A. B. (2001) 'Receptor-like kinases from Arabidopsis form a monophyletic gene family related to animal receptor kinases', Proceedings of the National Academy of Sciences . National Acad Sciences, 98(19), pp. 10763-10768.

Sinervo, B. et al. (2006) 'Genetic and maternal determinants of effective dispersal: the effect of sire genotype and size at birth in side-blotched lizards', The American Naturalist . The University of Chicago Press, 168(1), pp. 88-99.

Starrfelt, J. and Kokko, H. (2012) 'Bet-hedging — a triple trade-off between means, variances and correlations', Biological Reviews. Wiley Online Library, 87(3), pp. 742-755.

Steiner, B. L. et al. (2012) 'Distribution of bulbil-and seed-producing plants of Poa alpina (Poaceae) and their growth and reproduction in common gardens suggest adaptation to different elevations', American Journal of Botany, 99(12), pp. 2035-2044.

Tuljapurkar, S. (1990) Population dynamics in variable environments . Springer-Verlag Berlin Heidelberg.

Ummenhofer, C. C. and Meehl, G. A. (2017) 'Extreme weather and climate events with ecological relevance: a review', Philosophical Transactions of the Royal Society B: Biological Sciences . The Royal Society, 372(1723), p. 20160135.

Velchev, V. (1984) 'Plants', Red Data Book of the PR Bulgaria . Sophia: Publishing House Bulgarian Academy of Sciences, 1.

Venable, D. L. (1985) 'The evolutionary ecology of seed heteromorphism', The American Naturalist . University of Chicago Press, 126(5), pp. 577-595.

Venable, D. L. and Brown, J. S. (1993) 'The population-dynamic functions of seed dispersal', Vegetatio . Springer, 107(1), pp. 31-55.

Watson, R. T. et al. (1998) 'The regional impacts of climate change', IPCC, Geneva .

Wolff, J. O., Lundy, K. I. and Baccus, R. (1988) 'Dispersal, inbreeding avoidance and reproductive success in white-footed mice', Animal Behaviour. Elsevier, 36(2), pp. 456-465.

Figures 

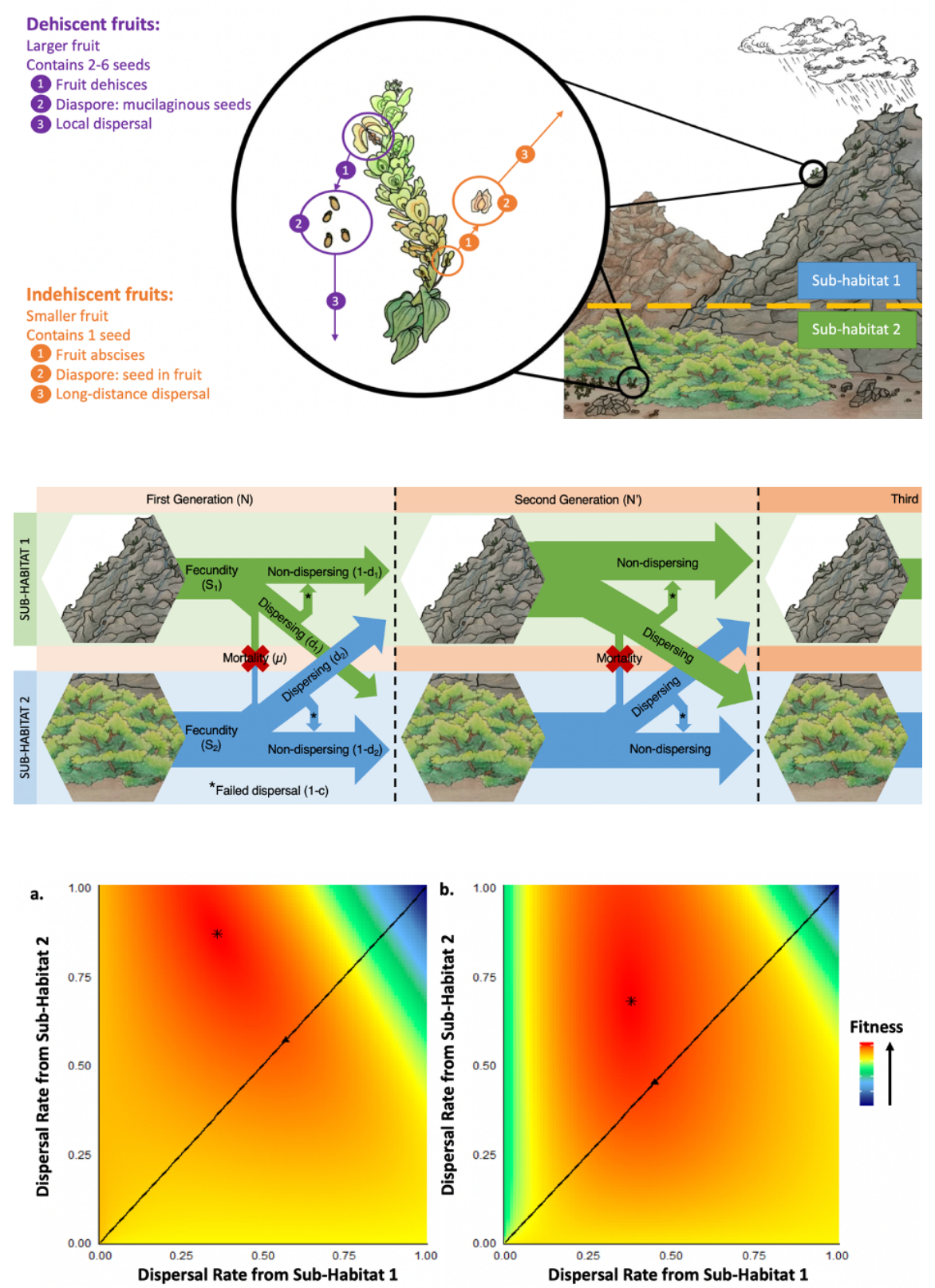

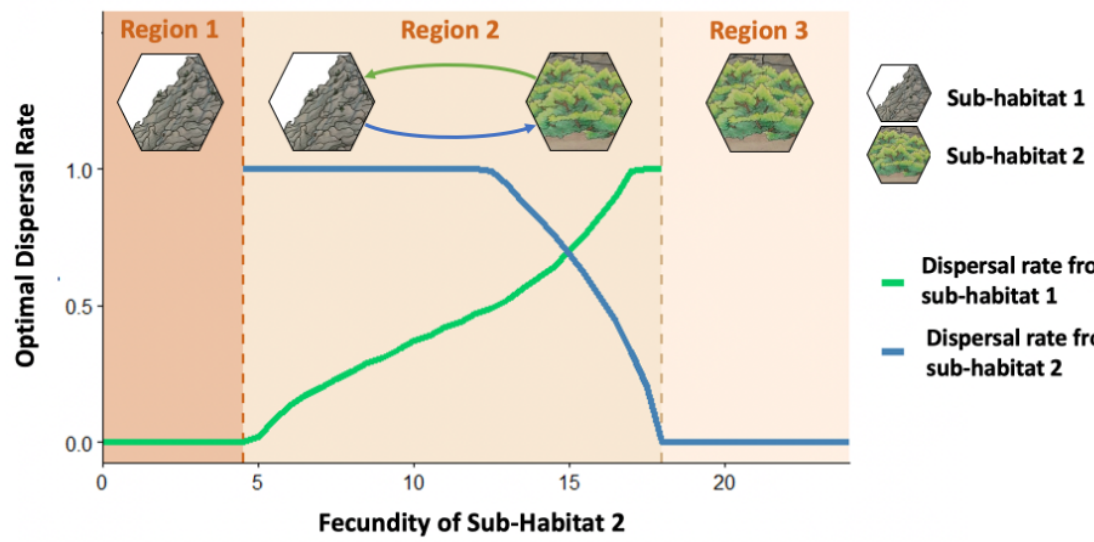

Dispersal rate from

sub-habitat 1

- Dispersal rate from sub-habitat 2

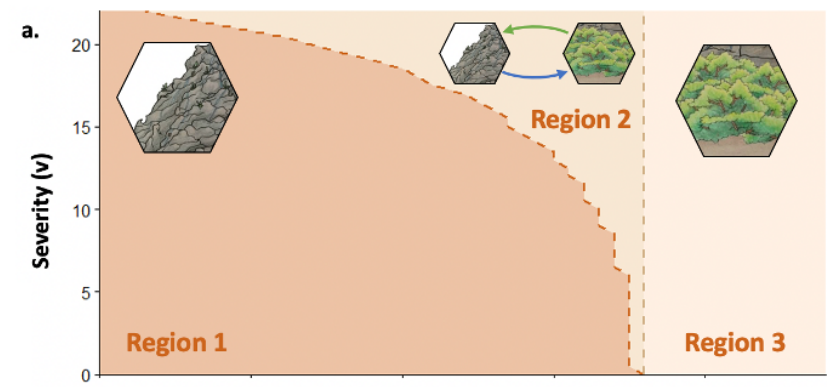

b.

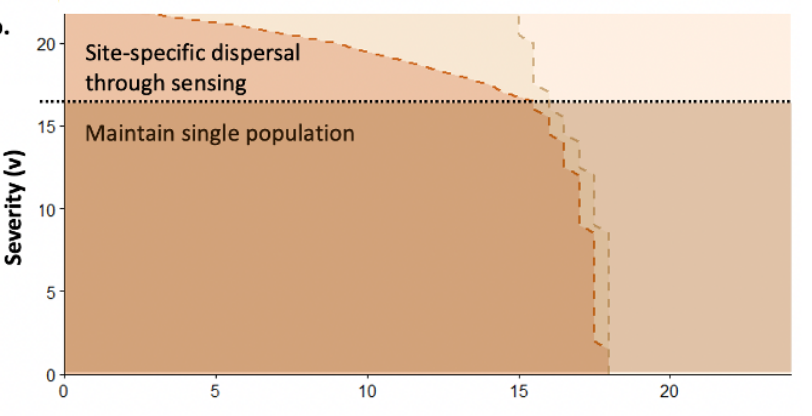

Sub-habitat 1

Sub-habitat 2

Fecundity of Sub-Habitat 2 